Internet Engineering Task Force (IETF)

J. Kelsey

Request for Comments: 5848

NIST

Category: Standards Track

J. Callas

ISSN : 2070-1721

PGP Corporation

A. Clemm

Cisco Systems

May 2010

\title{
Signed Syslog Messages
}

Abstract

This document describes a mechanism to add origin authentication, message integrity, replay resistance, message sequencing, and detection of missing messages to the transmitted syslog messages. This specification is intended to be used in conjunction with the work defined in RFC 5424, "The Syslog Protocol".

Status of This Memo

This is an Internet Standards Track document.

This document is a product of the Internet Engineering Task Force (IETF). It represents the consensus of the IETF community. It has received public review and has been approved for publication by the Internet Engineering Steering Group (IESG). Further information on Internet Standards is available in section 2 of RFC 5741.

Information about the current status of this document, any errata, and how to provide feedback on it may be obtained at http://www.rfc-editor.org/info/rfc5848.

Copyright Notice

Copyright (c) 2010 IETF Trust and the persons identified as the document authors. All rights reserved.

This document is subject to BCP 78 and the IETF Trust's Legal Provisions Relating to IETF Documents

(http://trustee.ietf.org/license-info) in effect on the date of publication of this document. Please review these documents carefully, as they describe your rights and restrictions with respect to this document. Code Components extracted from this document must include Simplified BSD License text as described in section $4 . e$ of the Trust Legal Provisions and are provided without warranty as described in the Simplified BSD License. 
This document may contain material from IETF Documents or IETF Contributions published or made publicly available before November 10, 2008. The person(s) controlling the copyright in some of this material may not have granted the IETF Trust the right to allow modifications of such material outside the IETF standards Process. Without obtaining an adequate license from the person(s) controlling the copyright in such materials, this document may not be modified outside the IETF standards Process, and derivative works of it may not be created outside the IETF Standards Process, except to format it for publication as an RFC or to translate it into languages other than English.

Table of Contents

1. Introduction . . . . . . . . . . . . . . . . . . . . . . 3

2. Conventions Used in This Document . . . . . . . . . . . . . . 5

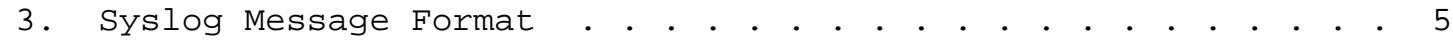

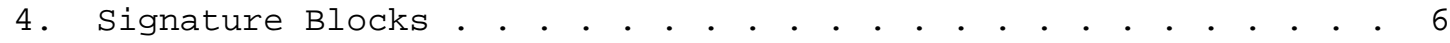
4.1. Syslog Messages Containing a Signature Block . . . . . . 7

4.2. Signature Block Format and Fields . • . . . . . . . . . . 7

4.2.1. Version . . . . . . . . . . . . . . . . . . . . . . 9

4.2.2. Reboot Session ID . . . . . . . . . . . . . . . . 10

4.2.3. Signature Group and Signature Priority . . . . . . 10

4.2.4. Global Block Counter. . . . . . . . . . . . . . . 13

4.2.5. First Message Number . . . . . . . . . . . . . 13

4.2.6. Count . . . . . . . . . . . . . . . . . . . . 14

4.2.7. Hash Block. . . . . . . . . . . . . . . . . . . 14

4.2.8. Signature . . . . . . . . . . . . . . . . . . 14

4.2.9. Example . . . . . . . . . . . . . . . . . . . 15

5. Payload and Certificate Blocks . • • . . . . . . . . . . 15

5.1. Preliminaries: Key Management and Distribution Issues . 15

5.2. Payload Block . . . . . . . . . . . . . . . . 16

5.2.1. Block Format and Fields . . . . . . . . . . . . . 16

5.2.2. Signer Authentication and Authorization . • . • . . 18

5.3. Certificate Block . . . . . . . . . . . . . . . . . 19

5.3.1. Syslog Messages Containing a Certificate Block . . . 19

5.3.2. Certificate Block Format and Fields . . . . . . . . 20

6. Redundancy and Flexibility . . . . . . . . . . . . . . . . . . 24

6.1. Configuration Parameters . . . . . . . . . . . . . . . 24

6.1.1. Configuration Parameters for Certificate Blocks . . . 24

6.1.2. Configuration Parameters for Signature Blocks . . . 26

6.2. Overlapping Signature Blocks • • • • • • • • • • • • • . . 27

7. Efficient Verification of Logs . . . . . . . . . . . . . . . 27

7.1. Offline Review of Logs . . . . . . . . . . . . . . . 28

7.2. Online Review of Logs . . . . . . . . . . . . . . . 29

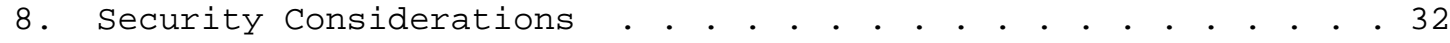

8.1. Cryptographic Constraints . . . . . . . . . . . . . . 32

8.2. Packet Parameters . . . . . . . . . . . . . . . . 33 
8.3. Message Authenticity
8.4.
8.5. .

\section{Introduction}

This document describes a mechanism, called syslog-sign in this document, that adds origin authentication, message integrity, replay resistance, message sequencing, and detection of missing messages to syslog. Essentially, this is accomplished by sending a special syslog message. The content of this syslog message is called a Signature Block. Each Signature Block contains, in effect, a detached signature on some number of previously sent messages. It is cryptographically signed and contains the hashes of previously sent syslog messages. The originator of syslog-sign messages is simply referred to as a "signer". The signer can be the same originator as the originator whose messages it signs, or it can be a separate originator.

While most implementations of syslog involve only a single originator and a single collector of each message, provisions need to be made to cover situations in which messages are sent to multiple collectors. This concerns, in particular, situations in which different messages from the same originator are sent to different collectors, which means that some messages are sent to some collectors but not to others. The required differentiation of messages is generally performed based on the Priority value of the individual messages. For example, messages from any Facility with a severity value of 3 , 2, 1, or 0 may be sent to one collector while all messages of Facilities 4, 10, 13, and 14 may be sent to another collector. Appropriate syslog-sign messages must be kept with their proper syslog messages. To address this, syslog-sign uses a Signature Group. A Signature Group identifies a group of messages that are all 
kept together for signing purposes by the signer. A Signature Block always belongs to exactly one Signature Group and always signs messages belonging only to that Signature Group.

Additionally, a signer sends Certificate Blocks to provide key management information between the signer and the collector. A Certificate Block has a field to denote the type of key material which may be such things as a Public Key Infrastructure using X.509 (PKIX) certificate, an OpenPGP (Pretty Good Privacy) certificate, or even an indication that a key had been pre-distributed. In the cases of certificates being sent, the certificates may have to be split across multiple Certificate Blocks carried in separate messages.

It is possible that the same host contains multiple signers that each use their own keys to sign syslog messages. In this case, each signer sends its own Certificate Block and Signature Blocks. Furthermore, each signer defines its own Signature Groups. Each signer on a given host needs to use a distinct combination of APPNAME, and PROCID for its Signature Block and Certificate Block message. (This implies that the combination of HOSTNAME, APP-NAME, and PROCID uniquely distinguishes originators of syslog-sign messages across hosts, provided that the signers use a unique HOSTNAME.)

The collector may verify that the hash of each received message matches the signed hash contained in the corresponding Signature Block. A collector may process these Signature Blocks as they arrive, building an authenticated log file. Alternatively, it may store all the log messages in the order they were received. This allows a network operator to authenticate the log file at the time the logs are reviewed.

The process of signing works as long as the collector accepts the syslog messages, the Certificate Blocks and the Signature Blocks. Once that is done, the process is complete. After that, anyone can go back, find the key material, and validate the received messages using the information in the Signature Blocks. Finding the key material is very easily done with Key Blob Types C, P, and K (see Section 4.2) since the public key is in the Payload Block. If Key Blob Types $\mathrm{N}$ or $\mathrm{U}$ are used, some poking around may be required to find the key material. The only way to have a vendor-specific implementation is through $\mathrm{N}$ or $\mathrm{U}$; however, also in that case, the key material will have to be available in some form which could be used by implementations of other vendors.

Because the mechanism that is described in this specification uses the concept of STRUCTURED-DATA elements defined in [RFC5424], compliant implementations of this specification MUST also implement [RFC5424]. It is conceivable that the concepts underlying this 
specification could also be used in conjunction with other messagedelivery mechanisms. Designers of other efforts to define event notification mechanisms are therefore encouraged to consider this specification in their designs.

2. Conventions Used in This Document

The key words "MUST", "MUST NOT", "REQUIRED", "SHALL", "SHALL NOT", "SHOULD", "SHOULD NOT", "RECOMMENDED", "MAY", and "OPTIONAL" in this document are to be interpreted as described in [RFC2119].

3. Syslog Message Format

This specification is intended to be used in conjunction with the syslog protocol as defined in [RFC5424]. The syslog protocol therefore MUST be supported by implementations of this specification.

Because the originator generating the Signature Block message, also simply referred to as "signer", signs each message in its entirety, the messages MUST NOT be changed in transit. By the same token, the syslog-sign messages MUST NOT be changed in transit. One of the effects of such behavior, including message alteration by relays, would be to render any signing invalid and hence make the mechanism useless. Likewise, any truncation of messages that occurs between sending and receiving renders the mechanism useless. For this reason, syslog signer and collector implementations implementing this specification MUST support messages of up to and including 2048 octets in length, in order to minimize the chance of truncation. While syslog signer and collector implementations MAY support messages with a length longer than 2048 octets, implementers need to be aware that any message truncations that occur render the mechanism useless. In such cases, it is up to the operator to ensure that the syslog messages can be received properly and can be validated.

[RFC5426] recommends using the Transport Layer Security (TLS) transport and deliberately constrains the use of UDP. UDP is NOT RECOMMENDED for use with signed syslog because its recommended payload size of 480 octets is too restrictive for the purposes of syslog-sign. A 480-octet Signature Block could sign only 9 normal messages, meaning that at a significant proportion of messages would be Signature Block messages. The 480-octet limitation is primarily geared towards small embedded systems with significant resource constraints that, because of those constraints, would not implement syslog-sign in the first place. In addition, the use of UDP is geared towards syslog messages that are primarily intended for troubleshooting, a very different purpose from the application targeted by syslog-sign. Where syslog UDP transport is used, it is the responsibility of operators to ensure that network paths are 
configured in a way that messages of sufficient length (up to and including 2048 octets) can be properly delivered.

This specification uses the syslog message format described in [RFC5424]. Along with other fields, that document describes the concept of Structured Data (SD). Structured Data is defined in terms of SD ELEMENTS (SDES). An SDE consists of a name and a set of parameter name-value pairs. The SDE name is referred to as SD-ID. The name-value pairs are referred to as SD-PARAM, or SD Parameters, with the name constituting the SD-PARAM-NAME, and the value constituting the SD-PARAM-VALUE.

The syslog messages defined in this document carry the data that is associated with Signature Blocks and Certificate Blocks as Structured Data. For this purpose, the special syslog messages defined in this document include definitions of SDEs to convey parameters that relate to the signing of syslog messages. The MSG part of the syslog messages defined in this document sHould simply be empty -- the content of the messages is not intended for interpretation by humans but by applications that use those messages to build an authenticated $\log$.

Because the syslog messages defined in this document adhere to the format described in [RFC5424], they identify the machine that originates the syslog message in the HOSTNAME field. Therefore, the Signature Block and Certificate Block data do not need to include any additional parameter to identify the machine that originates the message.

In addition, several signers MAY sign messages on a single host independently of each other, each using their own Signature Groups. In that case, each unique signer is distinguished by the combination of APP-NAME and PROCID. (By the same token, the same message might be signed by multiple signers.) Each unique signer MUST have a unique APP-NAME and PROCID on each host. (This implies that the combination of HOSTNAME, APP-NAME and PROCID uniquely distinguishes the originator of syslog-sign messages, provided that the signers use a unique HOSTNAME.) A Signature Block message MUST use the same combination of HOSTNAME, APP-NAME, and PROC-ID that was used to send the corresponding Certificate Block messages containing the Payload Block.

\section{Signature Blocks}

This section describes the format of the Signature Block and the fields used within the Signature Block, as well as the syslog messages used to carry the Signature Block. 


\subsection{Syslog Messages Containing a Signature Block}

There is a need to distinguish the Signature Block itself from the syslog message that is used to carry a Signature Block. Signature Blocks MUST be encompassed within completely formed syslog messages. Syslog messages that contain a signature Block are also referred to as Signature Block messages.

A Signature Block message is identified by the presence of an SD ELEMENT with an SD-ID with the value "ssign". In addition, a Signature Block message MUST contain valid APP-NAME, PROCID, and MSGID fields to be compliant with [RFC5424]. This specification does not mandate particular values for these fields; however, for consistency, a signer MUST use the same values for APP-NAME, PROCID, and MSGID fields for every Signature Block message that is sent, whichever values are chosen. It MUST also use the same value for its HOSTNAME field. To allow for the possibility of multiple signers per host, the combination of APP-NAME and PROCID MUST be unique for each such signer on any given host. If a signer daemon is restarted, it MAY use a new PROCID for what is otherwise the same signer but MUST continue to use the same APP-NAME. If it uses a new PROCID, it MUST send a new Payload Block using Certificate Block messages that use the same new PROCID (and the same APP-NAME). It is RECOMMENDED (but not required) to use 110 as value for the PRI field, corresponding to facility 13 (log audit) and severity 6 (informational). The Signature Block is carried as Structured Data within the Signature Block message, per the definitions that follow in the next section. A Signature Block message MAY carry other Structured Data besides the Structured Data of the Signature Block itself. The MSG part of a Signature Block message SHOULD be empty.

The syslog messages defined as part of syslog-sign themselves (Signature Block messages and Certificate Block messages) MUST NOT be signed by a Signature Block. Collectors that implement syslog-sign know to distinguish syslog messages that are associated with syslogsign from those that are subjected to signing and process them differently. The intent of syslog-sign is to sign a stream of syslog messages, not to alter it.

\subsection{Signature Block Format and Fields}

The content of a Signature Block message is the Signature Block itself. The Signature Block MUST be encoded as an SD ELEMENT, as defined in [RFC5424].

The SD-ID MUST have the value of "ssign". 
The SDE contains the fields of the Signature Block encoded as SD Parameters, as specified in the following. The Signature Block is composed of the following fields. The value of each field MUST be printable ASCII, and any binary values MUST be base64 encoded, as defined in [RFC4648].

\begin{tabular}{|c|c|c|}
\hline Field & SD-PARAM-NAME & Size in octets \\
\hline----- & ------------- & $----\quad--\quad------$ \\
\hline Version & VER & 4 \\
\hline Reboot Session ID & RSID & $1-10$ \\
\hline Signature Group & SG & 1 \\
\hline Signature Priority & SPRI & $1-3$ \\
\hline Global Block Counter & GBC & $1-10$ \\
\hline First Message Number & FMN & $1-10$ \\
\hline Count & $\mathrm{CNT}$ & $1-2$ \\
\hline Hash Block & $\mathrm{HB}$ & $\begin{array}{c}\text { variable, size of hash } \\
\text { times the number of hashes } \\
\text { (base64 encoded binary) }\end{array}$ \\
\hline Signature & SIGN & $\begin{array}{l}\text { variable } \\
\text { (base64 encoded binary) }\end{array}$ \\
\hline
\end{tabular}

The fields MUST be provided in the order listed. Each SD parameter MUST occur once and only once in the Signature Block. New SD parameters MUST NOT be added unless a new Version of the protocol is defined. (Implementations that wish to add proprietary extensions will need to define a separate SD ELEMENT.) A Signature Block is accordingly encoded as follows, where $\mathrm{xxx}$ denotes a placeholder for the particular values:

[ssign VER="xxx" RSID=" $x x x " S G=" x x x "$ SPRI=" $x x x "$ GBC=" $x x x "$ FMN=" $x x x "$ $\mathrm{CNT}=$ "xxx" HB="xxx" SIGN=" $\mathrm{x} x \mathrm{x} "$ ]

Values of the fields constitute SD parameter values and are hence enclosed in quotes, per [RFC5424]. The fields are separated by single spaces and are described in the subsequent subsections. 


\subsubsection{Version}

The Version field is an alphanumeric value that has a length of 4 octets, which may include leading zeroes. The first 2 octets and the last octet contain a decimal character in the range of "0" to "9", whereas the third octet contains an alphanumeric character in the range of "0" to "9", "a" to "z", or "A" to "Z". The value in this field specifies the version of the syslog-sign protocol. This is extensible to allow for different hash algorithms and signature schemes to be used in the future. The value of this field is the grouping of the protocol version (2 octets), the hash algorithm (1 octet), and the signature scheme (1 octet).

Protocol Version - 2 octets, with "01" as the value for the protocol version that is described in this document.

Hash Algorithm - 1 octet, where, in conjunction with Protocol Version 01, a value of "1" denotes SHA1 and a value of "2" denotes SHA256, as defined in [FIPS.180-2.2002]. (This is the octet that can have a value of not just "0" to "9" but also "a" to "z" and "A" to "Z".)

Signature Scheme - 1 octet, where, in conjunction with Protocol Version 01, a value of "1" denotes OpenPGP DSA, defined in [RFC4880] and [FIPS.186-2.2000].

The version, hash algorithm, and signature scheme defined in this document would accordingly be represented as "0111" (if SHA1 is used as Hash Algorithm) and "0121" (if SHA256 is used as Hash Algorithm), respectively (without the quotation marks).

The values of the Hash Algorithm and Signature Scheme are defined relative to the Protocol Version. If the single-octet representation of the values for Hash Algorithm and Signature Scheme were to ever represent a limitation, this limitation could be overcome by defining a new Protocol Version with additional Hash Algorithms and/or Signature Schemes, and having implementations support both Protocol Versions concurrently.

As long as the sender and receiver are both adhering to [RFC5424], the prerequisites are in place so that signed messages can be received by the receiver and validated with a Signature Block. To ensure immediate validation of received messages, all implementations MUST support SHA1, and SHA256 SHOULD be supported. 


\subsubsection{Reboot Session ID}

The Reboot Session ID is a decimal value that has a length between 1 and 10 octets. The acceptable values for this are between 0 and 9999999999. Leading zeroes MUST be omitted.

A Reboot Session ID is expected to strictly monotonically increase (i.e., to never repeat or decrease) whenever a signer reboots in order to allow collectors to distinguish messages and message signatures across reboots. There are several ways in which this may be accomplished. In one way, the Reboot Session ID may increase by 1, starting with a value of 1. Note that in this case, a signer is required to retain the previous Reboot Session ID across reboots. In another way, a value of the Unix time (number of seconds since 1 January 1970) may be used. Implementers of this method need to beware of the possibility of multiple reboots occurring within a single second. Implementers need to also beware of the year 2038 problem, which will cause the 32-bit representation of Unix time to wrap in the year 2038. In yet another way, implementations where the Simple Network Management Protocol (SNMP) engine and the signer always reboot at the same time might consider using the snmpEngineBoots value as a source for this counter as defined in [RFC3414].

In cases where a signer is not able to guarantee that the Reboot Session ID is always increased after a reboot, the Reboot session ID MUST always be set to a value of 0 . If the value can no longer be increased (e.g., because it reaches 9999999999), it SHoulD be reset to a value of 1 . Implementations SHOULD ensure that such a reset does not go undetected, for example, by requesting operator acknowledgment when a reset is performed upon reboot. (Operator acknowledgment may not be possible in all situations, e.g., in the case of embedded devices.)

If a reboot of a signer takes place, Signature Block messages MAY use a new PROCID. However, Signature Block messages of the same signer MUST continue to use the same HOSTNAME, APP-NAME, and MSGID.

\subsubsection{Signature Group and Signature Priority}

The SG parameter may take any value from 0-3 inclusive. The SPRI parameter may take any value from 0-191 inclusive. These fields taken together allow network administrators to associate groupings of syslog messages with appropriate Signature Blocks and Certificate Blocks. Groupings of syslog messages that are signed together are also called Signature Groups. A Signature Block contains only hashes of those syslog messages that are part of the same Signature Group. 
For example, in some cases, network administrators might have originators send syslog messages of Facilities 0 through 15 to one collector and those with Facilities 16 through 23 to another. In such cases, associated Signature Blocks should likely be sent to the corresponding collectors as well, signing the syslog messages that are intended for each collector separately. This way, each collector receives Signature Blocks for all syslog messages that it receives, and only for those. The ability to associate different categories of syslog messages with different Signature Groups, signed in separate Signature Blocks, provides administrators with flexibility in this regard.

Syslog-sign provides four options for handling Signature Groups, linking them with PRI values so they may be routed to the destination commensurate with the corresponding syslog messages. In all cases, no more than 192 distinct Signature Groups (0-191) are permitted.

The Signature Group to which a Signature Block pertains is indicated by the Signature Priority (SPRI) field. The Signature Group (SG) field indicates how to interpret the Signature Priority field. (Note that the SG field does not indicate the Signature Group itself, as its name might suggest.) The SG field can have one of the following values:

a. "0" -- There is only one Signature Group. In this case, the administrators want all Signature Blocks to be sent to a single destination; in all likelihood, all of the syslog messages will also be going to that same destination. Signature Blocks contain signatures for all messages regardless of their PRI value. This means that, in effect, the Signature Block's SPRI value can be ignored. However, it is RECOMMENDED that a single SPRI value be used for all Signature Blocks. Furthermore, it is RECOMMENDED to set that value to the same value as the PRI field of the Signature Block message. This way, the PRI of the Signature Block message matches the SPRI of the Signature Block that it contains.

b. "1" -- Each PRI value is associated with its own Signature Group. Signature Blocks for a given Signature Group have SPRI = PRI for that Signature Group. In other words, the SPRI of the Signature Block matches the PRI value of the syslog messages that are part of the Signature Group and hence signed by the Signature Block. An SG value of 1 can, for example, be used when the administrator of a signer does not know where any of the syslog messages will ultimately go but anticipates that messages with different PRI values will be collected and processed separately. Having a Signature Group per PRI value provides administrators with a large degree of flexibility with regard to how to divide up the 
processing of syslog messages and their signatures after they are received, at the same time allowing Signature Blocks to follow the corresponding syslog messages to their eventual destination.

c. "2" -- Each Signature Group contains a range of PRI values. Signature Groups are assigned sequentially. A Signature Block for a given Signature Group has its own SPRI value denoting the highest PRI value of syslog messages in that Signature Group. The lowest PRI value of syslog messages in that Signature Group will be 1 larger than the SPRI value of the previous Signature Group or "O" in case there is no other Signature Group with a lower SPRI value. The specific Signature Groups and ranges they are associated with are subject to configuration by a system administrator.

d. "3" -- Signature Groups are not assigned with any of the above relationships to PRI values of the syslog messages they sign. Instead, another scheme is used, which is outside the scope of this specification. There has to be some predefined arrangement between the originator and the intended collectors as to which syslog messages are to be included in which Signature Group, requiring configuration by a system administrator. This also provides administrators with the flexibility to group syslog messages into Signature Groups according to criteria that are not tied to the PRI value. Note that this option is not intended for deployments that lack such an arrangement, as in those cases a collector could misinterpret the intended meaning of the Signature Group. A collector that receives Signature Block messages of a Signature Group of whose scheme it is not aware SHOULD bring this fact to the attention of the system administrator. The particular mechanism used for that is implementation-specific and outside the scope of this specification.

One reasonable way to configure some installations is to have only one Signature Group, indicated with $\mathrm{SG}=0$, and have the signer send a copy of each Signature Block to each collector. In that case, collectors that are not configured to receive every syslog message will still receive signatures for every message, even ones they are not supposed to receive. While the collector will not be able to detect gaps in the messages (because the presence of a signature of a message that is missing does not tell the collector whether or not the corresponding message would be of the collector's concern), it does allow all messages that do arrive at each collector to be put into the right order and to be verified. It also allows each collector to detect duplicates. Likewise, configuring only one 
Signature Group can be a reasonable way to configure installations that involve relay chains, where one or more interim relays may or may not relay all messages to the same destination.

\subsubsection{Global Block Counter}

The Global Block Counter is a decimal value representing the number of Signature Blocks sent by syslog-sign before the current one, in this reboot session. This takes at least 1 octet and at most 10 octets displayed as a decimal counter. The acceptable values for this are between 0 and 9999999999, starting with 0 . Leading zeroes MUST be omitted. If the value of the Global Block Counter has reached 9999999999 and the Reboot Session ID has a value other than 0 (indicating the fact that persistence of the Reboot Session ID is supported), then the Reboot Session ID MUST be incremented by 1 and the Global Block Counter resumes at 0 . When the Reboot Session ID is 0 (i.e., persistent Reboot Session IDs are not supported) and the Global Block Counter reaches its maximum value, then the Global Block counter is reset to 0 and the Reboot Session ID MUST remain at 0 .

Note that the Global Block Counter crosses Signature Groups; it allows one to roughly synchronize when two messages were sent, even though they went to different collectors and are part of different Signature Groups.

Because a reboot results in the start of a new reboot session, the signer MUST reset the Global Block Counter to 0 after a reboot occurs. Applications need to take into account the possibility that a reboot occurred when authenticating a log, and situations in which reboots occur frequently may result in losing the ability to verify the proper sequence in which messages were sent, hence jeopardizing the integrity of the $\log$.

\subsubsection{First Message Number}

This is a decimal value between 1 and 10 octets, with leading zeroes omitted. It contains the unique message number within this signature Group of the first message whose hash appears in this block. The very first message of the reboot session is numbered "1". This implies that when the Reboot Session ID increases, the message number is reset to 1 .

For example, if this Signature Group has processed 1000 messages so far and message number 1001 is the first message whose hash appears in this Signature Block, then this field contains 1001 . The message number is relative to the Signature Group to which it belongs; hence, a message number does not identify a message beyond its signature Group. 
Should the message number reach 9999999999 within the same reboot session and Signature Group, the message number subsequently restarts at 1. In such an event, the Global Block Counter will be vastly different between two occurrences of the same message number.

4.2.6. Count

The count is a 1 - or 2-octet field that indicates the number of message hashes to follow. The valid values for this field are 1 through 99. The number of hashes included in the Signature Block MUST be chosen such that the length of the resulting syslog message does not exceed the maximum permissible syslog message length.

4.2.7. Hash Block

The hash block is a block of hashes, each separately encoded in base64. Each hash in the hash block is the hash of the entire syslog message represented by the hash, independent of the underlying transport. Hashes are ordered from left to right in the order of occurrence of the syslog messages that they represent. The space character is used to separate the hashes. Note, the hash block constitutes a single SD-PARAM; a Signature Block message MUST include all its hashes in a single hash block and MUST NOT spread its hashes across several hash blocks.

The "entire syslog message" refers to what is described as the syslog message excluding transport parts that are described in [RFC5425] and [RFC5426], and excluding other parts that may be defined in future transports. The hash value will be the result of the hashing algorithm run across the syslog message, starting with the "<" of the PRI portion of the header part of the message. The hash algorithm used and indicated by the Version field determines the size of each hash, but the size MUST NOT be shorter than 160 bits without the use of padding. It is base64 encoded as per [RFC4648].

The number of hashes in a hash block SHOULD be chosen such that the resulting Signature Block message does not exceed a length of 2048 octets in order to avoid the possibility that truncation occurs. When more hashes need to be sent than fit inside a Signature Block message, it is advisable to start a new Signature Block.

\subsubsection{Signature}

This is a digital signature, encoded in base64 per [RFC4648]. The signature is calculated over the completely formatted Signature Block message (starting from the first octet of PRI and continuing to the last octet of MSG, or STRUCTURED-DATA if MSG is not present), before the SIGN parameter (SD Parameter Name and the space before it 
[" SIGN"], "=", and the corresponding value) is added. (In other words, the digital signature is calculated over the whole message, with the "SIGN=value" portion removed.) For the OpenPGP DSA signature scheme, the value of the signature field contains the DSA values $r$ and $s$, encoded as two multiprecision integers (see [RFC4880], Sections 5.2.2 and 3.2), concatenated, and then encoded in base64 [RFC4648].

\subsubsection{Example}

An example of a Signature Block message is depicted below, broken into lines to fit publication rules. There is a space at the end of each line, with the exception of the last line, which ends with "]".

<110>1 2009-05-03T14:00:39.529966+02:00 host.example.org syslogd 2138 - [ssign VER="0111" RSID="1" SG="0" SPRI="0" GBC="2" FMN="1" $\mathrm{CNT}=" 7$ " HB="K6wzcombEvKJ+UTMcn9bPryAeaU= zrkDcIeaDluypaPCY8WWzwHpPok= zgrWOdpx16ADc7UmckyIFY53icE= XfopJ+S8/hODapiBBCgVQaLqBKg= J67gKMF1/OauTC20ibbydwIlJC8= M5GziVgB6KPY3ERU1HXdSi2vtdw= Wxd/IU7uG/ipEYT9xeqns fohyH0=" SIGN="AKBbX4 J7QkrwuwdbV7Taujk2 lvOf8gCgC62We1QYfnrNHz 7F zAvdySuMyfM=" ]

The message is of syslog-sign protocol version "01". It uses sHA1 as hash algorithm and an OpenPGP DSA signature scheme. Its reboot session ID is 1 . Its Signature Group is 0, which means that all syslog messages go to the same destination; its Signature Priority (which can effectively be ignored because all syslog messages will be signed regardless of their PRI value) is 0. Its Global Block Counter is 2. The first message number is 1 ; the message contains 7 message hashes.

5. Payload and Certificate Blocks

Certificate Blocks and Payload Blocks provide key management for syslog-sign. Their purpose is to support key management that uses public key cryptosystems.

\subsection{Preliminaries: Key Management and Distribution Issues}

A Payload Block contains public-key-certificate information that is to be conveyed to the collector. A Payload Block is sent at the beginning of a new reboot session, carrying public key information in effect for the reboot session. However, a Payload Block is not sent directly, but in (one or more) fragments. Those fragments are termed Certificate Blocks. Therefore, signers send at least one Certificate Block at the beginning of a new reboot session. 
There are three key points to understand about Certificate Blocks:

a. They handle a variable-sized payload, fragmenting it if necessary and transmitting the fragments as legal syslog messages. This payload is built (as described below) at the beginning of a reboot session and is transmitted in pieces with each Certificate Block carrying a piece. There is exactly one Payload Block per reboot session.

b. The Certificate Blocks are digitally signed. The signer does not sign the Payload Block, but the signatures on the Certificate Blocks ensure its authenticity. Note that it may not even be possible to verify the signature on the Certificate Blocks without the information in the Payload Block; in this case, the Payload Block is reconstructed, the key is extracted, and then the Certificate Blocks are verified. (This is necessary even when the Payload Block carries a certificate, because some other fields of the Payload Block are not otherwise verified.) In practice, most installations keep the same public key over long periods of time, so that most of the time, it is easy to verify the signatures on the Certificate Blocks, and use the Payload Block to provide other useful per-session information.

c. The kind of Payload Block that is expected is determined by what kind of key material is on the collector that receives it. The signer and collector (or offline log viewer) both have some key material (such as a root public key or pre-distributed public key) and an acceptable value for the key Blob Type in the Payload Block, below. The collector or offline log viewer MUST NOT accept a Payload Block of the wrong type.

5.2. Payload Block

The Payload Block is built when a new reboot session is started. There is a one-to-one correspondence between reboot sessions and Payload Blocks. A signer creates a new Payload Block after each reboot. The Payload Block is used until the next reboot.

\subsubsection{Block Format and Fields}

A Payload Block MUST have the following fields:

a. Full local timestamp for the signer at the time the reboot session started. This must be in the timestamp format specified in [RFC5424] (essentially, timestamp format per [RFC3339] with some further restrictions). 
b. Key Blob Type, a one-octet field containing one of five values:

1. ' $\mathrm{C}$ ' - a PKIX certificate (per [RFC5280]).

2. ' $\mathrm{P}$ ' -- an OpenPGP KeyID and OpenPGP certificate (a Transferable Public Key as defined in [RFC4880], Section 11.1). The first 8 octets of the key blob field contain the OpenPGP KeyID (identifying which key or subkey inside the OpenPGP certificate is used), followed by the OpenPGP certificate itself.

3. ' $\mathrm{K}$ ' -- the public key whose corresponding private key is being used to sign these messages. For the OpenPGP DSA signature scheme, the key blob field contains the DSA prime p, DSA group order $q$, DSA group generator $g$, and DSA publickey value $y$, encoded as 4 multiprecision integers (see [RFC4880], Sections 5.5.2 and 3.2).

4. ' $\mathrm{N}$ ' -- no key information sent; key is pre-distributed.

5. ' U' -- installation-specific key exchange information.

c. The key blob, if any, base64 encoded per [RFC4648] and consisting of the raw key data.

The fields are separated by single space characters. Because a Payload Block is not carried in a syslog message directly, only the corresponding Certificate Blocks, it does not need to be encoded as an SD ELEMENT. The Payload Block does not contain a field that identifies the reboot session; instead, the reboot session can be inferred from the Reboot Session ID parameter of the Certificate Blocks that are used to carry the Payload Block.

To ensure that the sender and receiver have at least one common Key Blob Type, for immediate validation of received messages, all implementations MUST support Key Blob Type "C" (PKIX certificate). When a PKIX certificate is used ("C" Key Blob Type), it is the certificate specified in [RFC5280]. Per [RFC5425], syslog messages may be transported over the TLS protocol, even where there is no PKI. If that transport is used, then the device will already have a PKIX certificate, and it MAY use the private key associated with that certificate to sign messages. In the case where there is no PKI, the chain of trust of a PKIX certificate must still be established to meet conventional security requirements. The methods for doing this are described in [RFC5425]. 


\subsubsection{Signer Authentication and Authorization}

When the collector receives a Payload Block, it needs to determine whether the signatures are to be trusted. The following methods are in scope of this specification:

a. X.509 certification path validation: The collector is configured with one or more trust anchors (typically root certification Authority (CA) certificates), which allow it to verify a binding between the subject name and the public key. Certification path validation is performed as specified in [RFC5280].

If the HOSTNAME contains a Fully-Qualified Domain Name (FQDN) or an IP address, it is then compared against the certificate as described in [RFC5425], Section 5.2. Comparing other forms of HOSTNAMEs is beyond the scope of this specification.

Collectors SHOULD support this method. Note that due to message size restrictions, syslog-sign sends only the end-entity certificate in the Payload Block. Depending on the PKI deployment, the collector may need to obtain intermediate certificates by other means (for example, from a directory).

b. X.509 end-entity certificate matching: The collector is configured with information necessary to identify the valid endentity certificates of its valid peers, and for each peer, the HOSTNAME (s) it is authorized to use.

To ensure interoperability, collectors MUST support fingerprints of X.509 certificates as described below. Other methods MAY be supported.

Collectors MUST support Key Blob Type ' $C$ ', and configuring the list of valid peers using certificate fingerprints. The fingerprint is calculated and formatted as specified in [RFC5425], Section 4.2.2.

For each peer, the collector MUST support configuring a list of HOSTNAMEs that this peer is allowed to use either as FQDNs or IP addresses. Other forms of HOSTNAMEs are beyond the scope of this specification.

If the locally configured FQDN is an internationalized domain name, conforming implementations MUST convert it to the ASCII Compatible Encoding ( $\mathrm{ACE}$ ) format for performing comparisons as specified in section 7 of [RFC5280]. An exact case-insensitive string match MUST be supported, but the implementation MAY also 
support wildcards of any type ("*", regular expressions, etc.) in locally configured names.

Signer implementations MUST provide a means to generate a key pair and self-signed certificate in the case that a key pair and certificate are not available through another mechanism, and MUST make the certificate fingerprint available through a management interface.

c. OpenPGP V4 fingerprints: Like X.509 fingerprints, except Key Blob Type ' $\mathrm{P}$ ' is used, and the fingerprint is calculated as specified in [RFC4880], Section 12.2. When the fingerprint value is displayed or configured, each byte is represented in hexadecimal (using two uppercase ASCII characters), and space is added after every second byte. For example: "0830 2A52 2CD1 D712 6E76 6EEC 32A5 CAE1 03C8 4F6E".

Signers and collectors MAY support this method.

Other methods, such as "web of trust", are beyond the scope of this document.

\subsection{Certificate Block}

This section describes the format of the Certificate Block and the fields used within the Certificate Block, as well as the syslog messages used to carry Certificate Blocks.

\subsubsection{Syslog Messages Containing a Certificate Block}

Certificate Blocks are used to get the Payload Block to the collector. As with a Signature Block, each Certificate Block is carried in its own syslog message, called a Certificate Block message. In case separate collectors are associated with different Signature Groups, Certificate Block messages need to be sent to each collector.

Because certificates can legitimately be much longer than 2048 octets, the Payload Block can be split up into several pieces, with each Certificate Block carrying a piece of the Payload Block. Note that the signer MAY make the Certificate Blocks of any legal length (that is, any length that keeps the entire Certificate Block message within 2048 octets) that holds all the required fields. Software that processes Certificate Blocks MUST deal correctly with blocks of any legal length. The length of the fragment of the Payload Block that a Certificate Block carries MUST be at least one octet. The length SHOULD be chosen such that the length of the Certificate Block message does not exceed 2048 octets. 
A Certificate Block message is identified by the presence of an SD ELEMENT with an SD-ID with the value "ssign-cert". In addition, a Certificate Block message MUST contain valid APP-NAME, PROCID, and MSGID fields to be compliant with syslog protocol. Syslog-sign does not mandate particular values for these fields; however, for consistency, a signer MUST use the same value for APP-NAME, PROCID, and MSGID fields for every Certificate Block message, whichever values are chosen. It MUST also use the same value for its HOSTNAME field. To allow for the possibility of multiple signers per host, the combination of APP-NAME and PROCID MUST be unique for each such originator. If a signer daemon is restarted, it MAY use a new PROCID for what is otherwise the same signer. The combination of APP-NAME and PROCID MUST be the same that is used for Signature Block messages of the same signer; however, a different MSGID MAY be used for Signature Block and Certificate Block messages. It is RECOMMENDED to use 110 as the value for the PRI field, corresponding to facility 13 (log audit) and severity 6 (informational). The Certificate Block is carried as Structured Data within the Certificate Block message. A Certificate Block message MAY carry other Structured Data besides the Structured Data of the Certificate Block itself. The MSG part of a Certificate Block message SHOULD be empty.

5.3.2. Certificate Block Format and Fields

The contents of a Certificate Block message is the Certificate Block itself. Like a Signature Block, the Certificate Block is encoded as an SD ELEMENT. The SD-ID of the Certificate Block is "ssign-cert". The Certificate Block is composed of the following fields, each of which is encoded as an SD Parameter with parameter name as indicated. Each field must be printable ASCII, and any binary values are base64 encoded per [RFC4648]. 


$\begin{array}{lcc}\text { Field } & \text { SD-PARAM-NAME } & \text { Size in octets } \\ -1 & \text { VER } & 4 \\ \text { Version } & \text { RSID } & 1-10 \\ \text { Reboot Session ID } & \text { SG } & 1 \\ \text { Signature Group } & \text { SPRI } & 1-3 \\ \text { Signature Priority } & \text { TPBL } & 1-8 \\ \text { Total Payload Block Length } & \text { INDEX } & 1-8 \\ \text { Index into Payload Block } & \text { FLEN } & 1-4 \\ \text { Fragment Length } & \text { FRAG } & \text { (base64 encoded binary) } \\ \text { Payload Block Fragment } & \text { SIGN } & \text { variable } \\ \text { Signature } & \text { (base64 encoded binary) }\end{array}$

The fields MUST be provided in the order listed. New SD parameters MUST NOT be added unless a new Version of the protocol is defined. (Implementations that wish to add proprietary extensions will need to define a separate SD ELEMENT.) A Certificate Block is accordingly encoded as follows, where xxx denotes a placeholder for the particular values:

[ssign-cert VER="xxx" RSID="xxx" SG="xxx" SPRI="xxx" TPBL="xxx" INDEX $=" x \times x "$ FLEN=" $x x x " F R A G=" x x x " S I G N=" x x x "]$

Values of the fields constitute SD parameter values and are hence enclosed in quotes, per [RFC5424]. The fields are separated by single spaces and are described below. Each SD parameter MUST occur once and only once.

5.3.2.1. Version

The Version field is 4 octets in length. This field is identical in format and meaning to the Version field described in section 4.2.1.

\subsubsection{Reboot Session ID}

The Reboot Session ID is identical in format and meaning to the RSID field described in section 4.2.2. 


\subsubsection{Signature Group and Signature Priority}

The SIG field is identical in format and meaning to the SIG field described in section 4.2.3. The SPRI field is identical in format and meaning to the SPRI field described there.

A signer SHOULD send separate Certificate Block messages for each Signature Group. This ensures that each collector that is associated with a Signature Group will receive the necessary key material in the case that messages of different Signature Groups are sent to different collectors. Note that the signer needs to get the same Payload Block to each collector, as for any given signer there is a one-to-one relationship between Payload Block and Reboot Session across all Signature Groups. Deployments that wish to associate different key material (and hence different Payload Blocks) with different Signature Groups can use separate signers for that purpose, each distinguished by its own combination of HOSTNAME, APP-NAME, and PROCID.

\subsubsection{Total Payload Block Length}

The Total Payload Block Length is a value representing the total length of the Payload Block in octets, expressed as a decimal with 1 to 8 octets with leading zeroes omitted.

\subsubsection{Index into Payload Block}

This is a decimal value between 1 and 8 octets, with leading zeroes omitted. It contains the number of octets into the Payload Block at which this fragment starts. The first octet of the first fragment is numbered "1". (Note, it is not numbered "0".)

\subsubsection{Fragment Length}

The total length of this fragment expressed as a decimal integer with 1 to 4 octets with leading zeroes omitted. The fragment length must be at least 1 .

\subsubsection{Payload Block Fragment}

The Payload Block Fragment contains a fragment of the payload block. Its length must match the indicated fragment length.

\subsubsection{Signature}

This is a digital signature, encoded in base64, as per [RFC4648]. The Version field effectively specifies the original encoding of the signature. The signature is calculated over the completely formatted 
Certificate Block message, before the SIGN parameter is added (see Section 4.2.8). For the OpenPGP DSA signature scheme, the value of the signature field contains the DSA values $r$ and $s$, encoded as 2 multiprecision integers (see [RFC4880], Sections 5.2.2 and 3.2), concatenated, and then encoded in base64 [RFC4648].

\subsubsection{Example}

An example of a Certificate Block message is depicted below, broken into lines to fit publication rules. There are no spaces at the end of the lines that contain the key blob and the signature.

<110>1 2009-05-03T14:00:39.519307+02:00 host.example.org syslogd 2138 - [ssign-cert VER="0111" RSID="1" SG="0" SPRI="0" TPBL="587" INDEX="1" FLEN="587" FRAG="2009-05-03T14:00:39.519005+02:00 K BACsLMZ NCV2NUAwe 4RAeAnSQuvv2KS51SnHFAaWJNU2XVDYvW1L jmJgg4vKvQP o3HEOD+2hEkt $1 \mathrm{z}$ CXADe03u 5pmHoWy 5FGi yCbgl YxJkUJJrQqlTSS 6vID 9yh smEnh0 7w3pos xmb 4 qYo 0uWQr AAenBweVMlBgV3ZA5 IMA8xq81+i 8wCgkWJjCjfLar7s+0X3HVrRroyARv8EAIYoxofh 9m N8n821BTTuQnz 5hp 40 d6Z3UudKePu2di 5Mx3 GFel wnV0 Qh 5mS s 0YkuHJg 0mcXyUAoeYry 5X6482 fUxbm+gOHVmYSDtBmZEB8P TEt 80 s 8 aedWgKEt / E 4 dT+Hmod 4 omECLteLXxt SCTM gDXYC+bSBMjRRCaeWhHrYYdYBACCWMdTc12hRLJTn8LX99kv1 I 7 qwg ieyna 8GCJv/rEgC SSS 9E1qARM+h19KovIUOh 14VzBw3rK7v8Dlw/CJyYDd5kwSvCw jho21 LiReeS 90VPYuZF RC1B82Sub152zOqICAWs gd4myCCiZbWBsuJ8P O gtarFIpleNacCc60V3i 2Rg==" SIGN="AKAQEUi ipt gpd0 I KcXbuggGXH / dCdQCgdysrTBLUlbeGAQ4 vwrnLOqSL 7+c= " ]

The message is of syslog-sign protocol version "01". It uses SHAl as hash algorithm and an OpenPGP DSA signature scheme. Its reboot session ID is 1. Its Signature Group is 0; its Signature Priority is 0. The Total Payload Block Length is 587 octets. The index into the payload block is 1 (meaning this is the first fragment). The length of the fragment is 587 (meaning that the Certificate Block message contains the entire Payload Block). The Payload Block has the timestamp 2009-05-03T14:00:39.519005+02:00. The Key Blob Type is ' $\mathrm{K}$ ', meaning that it contains a public key whose corresponding private key is being used to sign these messages.

Note that the Certificate Block message in this example has a timestamp that is very close to the timestamp in the Payload Block. The fact that the timestamps are so close implies that this is the first Certificate Block message sent in this reboot session; additional Certificate Block messages can be sent later with a later timestamp, which will carry the same Payload Block that will still contain the same timestamp. 
6. Redundancy and Flexibility

As described in section 8.5 of [RFC5424], a transport sender may discard syslog messages. Likewise, when syslog messages are sent over unreliable transport, they can be lost in transit. However, if a collector does not receive Signature and Certificate Blocks, many messages may not be able to be verified. The signer is allowed to send Signature and Certificate Blocks multiple times. Sending Signature and Certificate Blocks multiple times provides redundancy with the intent to ensure that the collector or relay does get the Signature Blocks and in particular the Payload Block at some point in time. In the meantime, any online review of logs as described in Section 7.2 is delayed until the needed blocks are received. The collector MUST ignore duplicates of Signature Blocks and Certificate Blocks that it has already received and authenticated. In principle, the signer can change its redundancy level for any reason, without communicating this fact to the collector.

A signer that is also the originator of messages that it signs does not need to queue up other messages while sending redundant Certificate Block and Signature Block messages. It MAY send redundant Certificate Block messages even after Signature Block messages and regular syslog messages have been sent. By the same token, it MAY send redundant Signature Block messages even after newer syslog messages that are signed by a subsequent Signature Block have been sent, or even after a subsequent signature Block message.

In addition, the signer has flexibility in how many hashes to include within a Signature Block. It is legitimate for an originator to send short Signature Blocks to allow the collector to verify messages with minimal delay.

\subsection{Configuration Parameters}

Although the transport sender is not constrained in how it decides to send redundant Signature and Certificate Blocks, or even in whether it decides to send along multiple copies of normal syslog messages, we define some redundancy parameters below that may be useful in controlling redundant transmission from the transport sender to the transport receiver and that may be useful for administrators to configure.

\subsubsection{Configuration Parameters for Certificate Blocks}

Certificate Blocks are always sent at the beginning of a new reboot session. One technique to ensure reliable delivery (see Section 8.5) is to send multiple copies. This can be controlled by a "certInitialRepeat" parameter: 
certInitialRepeat $=$ number of times each Certificate Block should be sent before the first message is sent.

It is also useful to resend Certificate Blocks every now and then for long-lived reboot sessions. This can be controlled by the certResendDelay and certResendCount parameters:

certResendDelay = maximum time delay in seconds until resending the Certificate Block.

certResendCount = maximum number of other syslog messages to send until resending the Certificate Block.

In some cases, it may be desirable to allow for configuration of the transport sender such that Certificate Blocks are not sent at all after the first normal syslog message has been sent. This could be expressed by setting both certResendDelay and certResendCount to "0". However, configuring the transport sender to send redundant Certificate Blocks even after the first message, in particular when the UDP transport [RFC5426] is used, is RECOMMENDED.

In one set of circumstances, the receiver may receive a Certificate Block, some group of syslog messages, and some corresponding Signature Blocks. If the receiver reboots after that, then the conditions of recovery will vary depending upon the transport. For UDP [RFC5426], the receiver SHOULD continue to use the cached Certificate Block, but MUST validate the RSID value to make sure that it has the most current one. If the receiver cannot validate that it has the most current Certificate Block, then it MUST wait for a retransmission of the Certificate Block, which may be controlled by the certResendDelay and certResendCount parameters. It is up to the operators to ensure that Certificate Blocks are sent frequently enough to meet this set of circumstances.

For TLS transport [RFC5425], the sender MUST send a fresh Certificate Block when a session is established. This will keep the sender and receiver synchronized with the most current Certificate Block.

Implementations that support sending syslog messages of different Signature Groups to different collectors and which wish to offer very granular controls MAY allow the above parameters to be configured on a per Signature Group basis.

The choice of reasonable values in a given deployment depends on several factors, including the acceptable delay that may be incurred from the receipt of a syslog message until the corresponding Signature Block is received, whether UDP or TLS transport is used, and the available management bandwidth. The following might be a 
reasonable choice for a deployment in which reliability of underlying transport and of collector implementation are of little concern:

certInitialRepeat $=1$, certResendDelay $=1800$ seconds, certResendCount $=10000$

The following might be a reasonable choice for a deployment in which reliability of transmission over UDP transport could be an issue:

certInitialRepeat $=2$, certResendDelay $=300$ seconds, certResendCount $=1000$

\subsubsection{Configuration Parameters for Signature Blocks}

Verification of log messages involves a certain delay of time that is caused by the lag in time between the sending of the message itself and the corresponding Signature Block. The following configuration parameter can be useful to limit the time lag that will be incurred (note that the maximum message length may also force generating a Signature Block; see Sections 4.2.6 and 4.2.7):

sigMaxDelay = generate a new Signature Block if this many seconds have elapsed since the message with the First Message Number of the Signature Block was sent.

Retransmissions of Signature Blocks are not sent immediately after the original transmission, but slightly later. The following parameters control when those retransmissions are done:

sigNumberResends $=$ number of times a Signature Block is resent. (It is recommended to select a value of greater than "0" in particular when the UDP transport [RFC5426] is used.)

sigResendDelay = send the next retransmission when this many seconds have elapsed since the previous sending of this signature Block.

sigResendCount $=$ send the next retransmission when this many other syslog messages have been sent since the previous sending of this Signature Block.

The choice of reasonable values in a given deployment depends on several factors, including the acceptable delay that may be incurred from the receipt of a syslog message until the corresponding Signature Block is received so that the syslog message can be verified, the reliability of the underlying transport, and the available management bandwidth. The following might be a reasonable choice for a deployment where reliability of transport and collector 
are of little concern and where there is a need to have syslog messages generally signed within 5 minutes:

sigMaxDelay=300 seconds, sigNumberResends=2, sigResendDelay=300 seconds, sigResendCount $=500$

The following would be a reasonable choice for a deployment that needs to validate syslog messages typically within 60 seconds, but no more than 3 minutes after receipt:

sigMaxDelay=30 seconds, sigNumberResends=5, sigResendDelay=30 seconds, sigResendCount $=100$

\subsection{Overlapping Signature Blocks}

Notwithstanding the fact that the signer is not constrained in whether it decides to send redundant Signature Block messages, Signature Blocks SHOULD NOT overlap. This facilitates their processing by the receiving collector. This means that an originator of Signature Block messages, after having sent a first message with some First Message Number and a Count, SHOULD NOT send a second message with the same First Message Number but a different Count. It also means that an originator of Signature Block messages SHOULD NOT send a second message whose First Message Number is greater than the First Message Number, but smaller than the First Message Number plus the count indicated in the first message.

That said, the possibility of Signature Blocks that overlap does provide additional flexibility with regard to redundancy; it provides an additional option that may be desirable in some deployments. Therefore, collectors MUST be designed in a way that they can cope with overlapping Signature Blocks when confronted with them. The collector MUST ignore hashes of messages that it has already received and validated.

7. Efficient Verification of Logs

The logs secured with syslog-sign may be reviewed either online or offline. Online review is somewhat more complicated and computationally expensive, but not prohibitively so. This section outlines a method for online and a method for offline verification of logs that implementations MAY choose to implement to verify logs efficiently. Implementations MAY also choose to implement a different method; it is ultimately up to each implementation how to process the messages that it receives. 


\subsection{Offline Review of Logs}

When the collector stores logs to be reviewed later, they can be authenticated offline just before they are reviewed. Reviewing these logs offline is simple and relatively inexpensive in terms of resources used, so long as there is enough space available on the reviewing machine.

To do so, we first go through the stored log file. Each message in the log file is classified as a normal message, a Signature Block message, or a Certificate Block message. Signature Blocks and Certificate Blocks are then separated by signer (as identified by HOSTNAME, APP-NAME, PROCID), Reboot Session ID, and Signature Group, and stored in their own files. Normal messages are stored in a keyed file, indexed on their hash values. They are not separated by signer, as their (HOSTNAME, APP-NAME, PROCID) identifies the application that generated the message. The application that generated the message does not have to coincide with the signer.

For each signer, Reboot Session ID, and Signature Group, we then:

a. Sort the Certificate Block file by INDEX value, and check to see whether we have a set of Certificate Blocks that can reconstruct the Payload Block. If so, we reconstruct the Payload Block, verify any key-identifying information, and then use this to verify the signatures on the Certificate Blocks we have received. When this is done, we have verified the reboot session and key used for the rest of the process.

b. Sort the Signature Block file by First Message Number. We now create an authenticated log file, which consists of some header information and then a (sequence of message number, message text pairs). We next go through the Signature Block file. We initialize a cursor for the last message number processed with the number 0 . For each Signature Block in the file, we do the following:

1. Verify the signature on the Signature Block.

2. If the value of the First Message Number of the Signature Block is less than or equal to the last message number processed, skip the first (last message number processed minus First Message Number plus 1) hashes.

3. For each remaining hashed message in the Signature Block: a. Look up the hash value in the keyed message file. 
b. If the message is found, write (message number, message text) to the authenticated log file.

4. Set the last message number processed to the value of the First Message Number plus the Count of the Signature Block minus 1 .

5. Skip all other Signature Blocks with the same First Message Number unless one with a larger count is encountered.

The resulting authenticated log file contains all messages that have been authenticated. In addition, it implicitly indicates all gaps in the authenticated messages (specifically in the case when all messages of the same Signature Group are sent to the same collector), because their message numbers are missing.

One can see that, assuming sufficient space for building the keyed file, this whole process is linear in the number of messages (generally two seeks, one to write and the other to read, per normal message received), and $O(N$ lg $N)$ in the number of Signature Blocks. This estimate comes with two caveats: first, the Signature Blocks arrive very nearly in sorted order, and so can probably be sorted more cheaply on average than $O(N$ lg N) steps. Second, the signature verification on each Signature Block almost certainly is more expensive than the sorting step in practice. We have not discussed error-recovery, which may be necessary for the Certificate Blocks. In practice, a simple error-recovery strategy is probably enough: if the Payload Block is not valid, then we can just try alternate instances of each Certificate Block, if such are available, until we get the Payload Block right.

It is easy for an attacker to flood us with plausible-looking messages, Signature Blocks, and Certificate Blocks.

\subsection{Online Review of Logs}

Some collector implementations may need to monitor log messages in close to real time. This can be done with syslog-sign, though it is somewhat more complex than offline verification. This is done as follows :

a. We have an authenticated message file, into which we write (message number, message text) pairs that have been authenticated. We will assume that we are handling only one signer, Signature Group, and Reboot Session ID at any given time. (For the concurrent support of multiple signers, Signature Groups, and Reboot Session IDs, the same procedure is applied analogously to each. Signature Block messages and Certificate 
Block messages clearly indicate their respective signer, Signature Group, and Reboot Session ID.)

b. We have two data structures: A "Waiting for Signature" queue in which (arrival sequence, hash of message) pairs are kept in sorted order, and a "Waiting for Message" queue in which (message number, hash of message) pairs are kept in sorted order. In addition, we have a hash table that stores (message text, count) pairs indexed by hash value. In the hash table, count may be any number greater than zero; when count is zero, the entry in the hash table is cleared.

Note: The "Waiting for Signature" queue gets used in the normal case, when the signature arrives after the message itself. It holds messages that have been received but whose signature has yet to arrive. The "Waiting for Message" queue gets used in the case that messages are lost or misordered (either in the network or in relays). It holds signatures that have been received but whose corresponding messages have yet to arrive. Since a single Signature Block can cover only a limited number of messages (due to size restrictions), and massive reordering/delaying is rare, it is expected that both queues would be relatively small.

c. We must receive all the Certificate Blocks before any other processing can really be done. (This is why they are sent first.) Once that is done, any additional Certificate Block message that arrives is discarded. Any syslog messages or Signature Block messages that arrive before all Certificate Blocks have been received need to be buffered. Once all Certificate Blocks have been received, the messages in the buffer can be retrieved and processed as if they were just arriving.

d. Whenever a normal message arrives, we first check if its hash value is found in the "Waiting for Message" queue. If it is, we write the message number (from the "Waiting for Message" queue) and the message into the authenticated message file and remove the entry from the queue.

Otherwise, we add (arrival sequence, hash of message) to the "Waiting for Signature" queue. If our hash table already has an entry for the message's hash value, we increment its count by one; otherwise, we create a new entry with count $=1$.

If the "Waiting for Signature" message queue is full, we remove the oldest message from the queue. That message could not be validated close enough to real time. In order to update the hash table accordingly, we use that entry's hash to index the hash table. If that entry has count 1, we delete the entry from the 
hash table; otherwise, we decrement its count. By removing the message from the "Waiting for Signature" message queue without having actually received the message's signature, we make it impossible to authenticate the message should its signature arrive later. Implementers therefore need to ensure that queues are dimensioned sufficiently large to not expose the collector against Denial-of-Service (DoS) attacks that attempt to flood the collector with unsigned messages.

e. Whenever a Signature Block message arrives, we check its originator, (i.e., the signer) by way of HOSTNAME, APP-NAME, and PROCID, as well as its Signature Group and Reboot session ID to ensure it matches our Certificate Blocks. We then check to see whether the First Message Number value is too old to still be of interest, or if another Signature Block with that First Message Number and the same count or a greater count has already been received. If so, we discard the Signature Block. We then check the signature. Again, we discard the Signature Block if the signature is not valid.

Otherwise, we proceed with processing the hashes in the Signature Block. A Signature Block contains a sequence of hashes, each of which is associated with a message number, starting with the First Message Number for the first hash and incrementing by one for each subsequent hash. For each hash, we first check to see whether the message hash is in the hash table. If this is the case, it means that we have received the signature for a message that was received earlier, and we do the following:

1. We check if a message with the same message number is already in the authenticated message file. If that is the case, the signed hash is a duplicate and we discard it.

2. Otherwise (the signed hash is not a duplicate), we write the (message number, message text) into the authenticated message file. We also update the hash table accordingly, using that entry's hash to index the hash table. If that entry has Count 1, we delete the entry from the hash table; otherwise, we decrement its count.

Otherwise (the message hash is not in the hash table), we write the (message number, message hash) to the "Waiting for Message" queue.

If the "Waiting for Message" queue is full, we remove the oldest entry. In that case, a message that was signed by the signer could not be validated by the receiver, either because the message was lost or because the signature arrived way ahead of 
the actual message. By removing the entry from the "Waiting for Message" queue without having actually received the message, we make it impossible to authenticate the a legitimate message should that message still arrive later. Implementers need to ensure queues are dimensioned sufficiently large so that the chances of such a scenario actually occurring is minimized.

f. The result of this is a sequence of messages in the authenticated message file. Each message in the message file has been authenticated. The sequence is labeled with numbers showing the order in which the messages were originally transmitted.

One can see that this whole process is roughly linear in the number of messages, and also in the number of signature Blocks received. The process is susceptible to flooding attacks; an attacker can send enough normal messages that the messages roll off their queue before their Signature Blocks can be processed.

8. Security Considerations

Normal syslog event messages are unsigned and have most of the security attributes described in section 8 of [RFC5424]. This document also describes Certificate Blocks and Signature Blocks, which are signed syslog messages. The Signature Blocks contain signature information for previously sent syslog event messages. All of this information can be used to authenticate syslog messages and to minimize or obviate many of the security concerns described in [RFC5424].

The model for syslog-sign is a direct trust system where the certificate transferred is its own trust anchor. If a transport sender sends a stream of syslog messages that is signed using a certificate, the operator or application will transfer to the transport receiver the certificate that was used when signing. There is no need for a certificate chain.

\subsection{Cryptographic Constraints}

As with any technology involving cryptography, it is advisable to check the current literature to determine whether any algorithms used here have been found to be vulnerable to attack.

This specification uses Public Key Cryptography technologies. The proper party or parties have to control the private key portion of a public-private key pair. Any party that controls a private key can sign anything it pleases. 
Certain operations in this specification involve the use of random numbers. An appropriate entropy source SHOULD be used to generate these numbers. See [RFC4086] and [NIST800.90].

\subsection{Packet Parameters}

As a signer, it is advisable to avoid message lengths exceeding 2048 octets. Various problems might result if a signer were to send messages with a length greater than 2048 octets, because relays MAY truncate messages with lengths greater than 2048 octets, which would make it impossible for collectors to validate a hash of the packet. To increase the chance of interoperability, it tends to be best to be conservative with what you send but liberal in what you are able to receive.

Signers need to rigidly adhere to the RFC 5424 format when sending messages. If a collector receives a message that is not formatted properly, then it might drop it, or it may modify it while receiving it. (See Appendix A.2 of [RFC5424].) If that were to happen, the hash of the sent message would not match the hash of the received message.

Collectors are not to malfunction in the case that they receive malformed syslog messages or messages containing characters other than those specified in this document. In other words, they are to ignore such messages and continue working.

8.3. Message Authenticity

Syslog does not strongly associate the message with the message originator. That association is established by the collector upon verification of the Signature Block. Before a Signature Block is used to ascertain the authenticity of an event message, it might be received, stored, and reviewed by a person or automated parser. It is advisable not to assume a message is authentic until after a message has been validated by checking the contents of the Signature Block.

With the Signature Block checking, an attacker may only forge messages if he or she can compromise the private key of the true originator.

\subsection{Replaying}

Event messages might be recorded and replayed by an attacker. Using the information contained in the Signature Blocks, a reviewer can determine whether the received messages are the ones originally sent by an originator. The reviewer can also identify messages that have 
been replayed. Using a method for the verification of logs such as the one outlined in section 7, a replayed message can be detected by checking prior to writing a message to the authenticated log file whether the message is already contained in it.

\subsection{Reliable Delivery}

Event messages sent over UDP might be lost in transit. [RFC5425] can be used for the reliable delivery of syslog messages; however, it does not protect against loss of syslog messages at the application layer, for example, if the TCP connection or TLS session has been closed by the transport receiver for some reason. A reviewer can identify any messages sent by the originator but not received by the collector by reviewing the Signature Block information. In addition, the information in subsequent Signature Blocks allows a reviewer to determine whether any Signature Block messages were lost in transit.

\subsection{Sequenced Delivery}

Syslog messages delivered over UDP might not only be lost, but also arrive out of sequence. A reviewer can determine the original order of syslog messages and identify which messages were delivered out of order by examining the information in the Signature Block along with any timestamp information in the message.

\subsection{Message Integrity}

Syslog messages might be damaged in transit. A review of the information in the Signature Block determines whether the received message was the intended message sent by the originator. A damaged Signature Block or Certificate Block is evident because the collector will not be able to validate that it was signed by the signer.

\subsection{Message Observation}

Unless TLS is used as a secure transport [RFC5425], event messages, Certificate Blocks, and Signature Blocks are all sent in plaintext. This allows network administrators to read the message when sniffing the wire. However, this also allows an attacker to see the contents of event messages and perhaps to use that information for malicious purposes.

\subsection{Man-in-the-Middle Attacks}

It is conceivable that an attacker might intercept Certificate Block messages and insert its own Certificate information. In that case, the attacker would be able to receive event messages from the actual originator and then relay modified messages, insert new messages, or 
delete messages. It would then be able to construct a signature Block and sign it with its own private key. Network administrators need to verify that the key contained in the Payload Block is indeed the key being used on the actual signer. If that is the case, then this MITM attack will not succeed. Methods for establishing a chain of trust are also described in [RFC5425].

8.10. Denial of Service

An attacker might send invalid Signature Block messages to overwhelm the collector's processing capability and consume all available resources. For this reason, it can be appropriate to simply receive the Signature Block messages and process them only as time permits.

An attacker might also just overwhelm a collector by sending more messages to it than it can handle. Implementers are advised to consider features that minimize this threat, such as only accepting syslog messages from known IP addresses.

\subsection{Covert Channels}

Nothing in this protocol attempts to eliminate covert channels. In fact, just about every aspect of syslog messages lends itself to the conveyance of covert signals. For example, a collusionist could send odd and even PRI values to indicate Morse Code dashes and dots.

9. IANA Considerations

\subsection{Structured Data and Syslog Messages}

With regard to [RFC5424], IANA has added the following values (with each parameter listed as mandatory) to the registry titled "syslog Structured Data ID Values": 


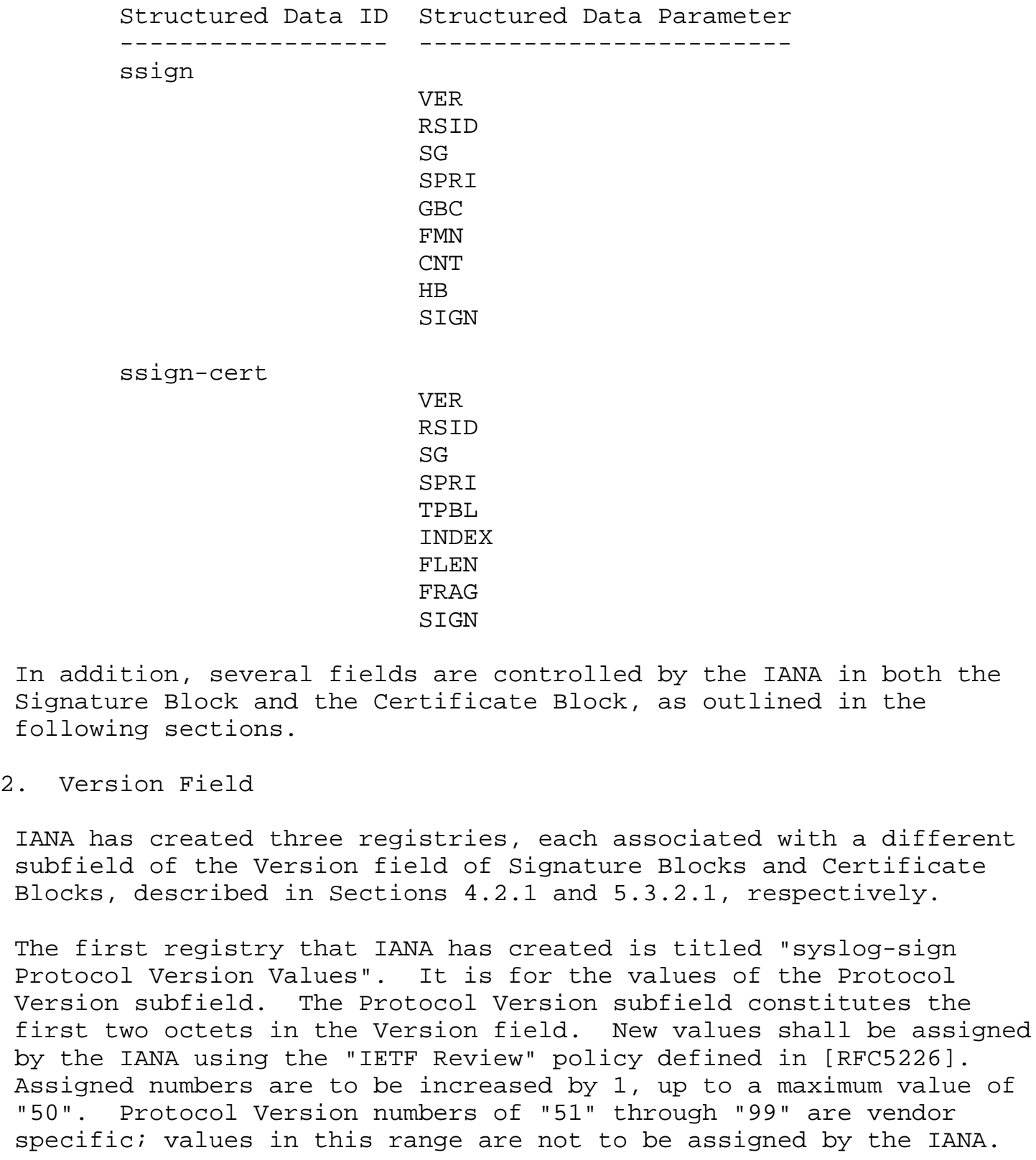

In addition, several fields are controlled by the IANA in both the Signature Block and the Certificate Block, as outlined in the

IANA has created three registries, each associated with a different subfield of the Version field of Signature Blocks and Certificate Blocks, described in Sections 4.2.1 and 5.3.2.1, respectively.

The first registry that IANA has created is titled "syslog-sign Protocol Version Values". It is for the values of the Protocol Version subfield. The Protocol Version subfield constitutes the first two octets in the Version field. New values shall be assigned by the IANA using the "IETF Review" policy defined in [RFC5226]. Assigned numbers are to be increased by 1, up to a maximum value of "50". Protocol Version numbers of "51" through "99" are vendor specific; values in this range are not to be assigned by the IANA. following sections.

\subsection{Version Field}


IANA has registered the Protocol Version values shown below.

$\begin{array}{ll}\text { Value } & \text { Protocol Version } \\ ----- & ------------ \\ 00 & \text { Reserved } \\ 01 & \text { Defined in RFC 5848 }\end{array}$

The second registry that IANA has created is titled "syslog-sign Hash Algorithm Values". It is for the values of the Hash Algorithm subfield. The Hash Algorithm subfield constitutes the third octet in the Version field Signature Blocks and Certificate Blocks. New values shall be assigned by the IANA using the "IETF Review" policy defined in [RFC5226]. Assigned values are to be increased sequentially, first up to a maximum value of "9", then from "a" to "z", then from "A" to "Z". The values are registered relative to the Protocol Version. This means that the same Hash Algorithm value can be reserved for different Protocol Versions, possibly referring to a different hash algorithm each time. This makes it possible to deal with future scenarios in which the single octet representation becomes a limitation, as more Hash Algorithms can be supported by defining additional Protocol Versions that implementations might support concurrently.

IANA has registered the Hash Algorithm values shown below.

$\begin{array}{lll}\text { Value } & \text { Protocol Version } & \text { Hash Algorithm } \\ ----- & ------------- & ------------ \\ 0 & 01 & \text { Reserved } \\ 1 & 01 & \text { SHA1 } \\ 2 & 01 & \text { SHA256 }\end{array}$

The third registry that IANA has created is titled "syslog-sign Signature Scheme Values". It is for the values of the Signature Scheme subfield. The Signature Scheme subfield constitutes the fourth octet in the Version field of Signature Blocks and Certificate Blocks. New values shall be assigned by the IANA using the "IETF Review" policy defined in [RFC5226]. Assigned values are to be increased by 1, up to a maximum value of "9". This means that the same Signature Scheme value can be reserved for different Protocol Versions, possibly in each case referring to a different signature Scheme each time. This makes it possible to deal with future scenarios in which the single octet representation becomes a limitation, as more Signature schemes can be supported by defining additional Protocol Versions that implementations might support concurrently. 
IANA has registered the Signature Scheme values shown below.

$\begin{array}{lll}\text { Value } & \text { Protocol Version } & \text { Signature Scheme } \\ ----- & ------------ & ----------- \\ 0 & 01 & \text { Reserved } \\ 1 & 01 & \text { OpenPGP DSA }\end{array}$

9.3. SG Field

IANA has created a registry titled "syslog-sign SG Field Values". It is for values of the SG Field as defined in Section 4.2.3. New values shall be assigned by the IANA using the "IETF Review" policy defined in [RFC5226]. Assigned values are to be incremented by 1, up to a maximum value of "7". Values "8" and "9" shall be left as vendor specific and shall not be assigned by the IANA.

IANA has registered the SG Field values shown below.

$\begin{array}{ll}\text { Value } & \text { Meaning } \\ 0 & -----\end{array}$

9.4. Key Blob Type

IANA has created a registry titled "syslog-sign Key Blob Type Values". It is to register one-character identifiers for the Key Blob Type, per section 5.2. New values shall be assigned by the IANA using the "IETF Review" policy defined in [RFC5226]. Uppercase letters may be assigned as values. Lowercase letters are left as vendor specific and shall not be assigned by the IANA.

IANA has registered the Key Blob Type values shown below.

$\begin{array}{ll}\text { Value } & \text { Key Blob Type } \\ ---- & -------- \\ \mathrm{C} & \text { a PKIX certificate } \\ \mathrm{P} & \text { an OpenPGP certificate } \\ \mathrm{K} & \text { the public key whose corresponding private key is } \\ & \text { used to sign the messages } \\ \mathrm{N} & \text { no key information sent, key is pre-distributed } \\ \mathrm{U} & \text { installation-specific key exchange information }\end{array}$




\section{Acknowledgements}

The authors wish to thank the current Chairs of the Syslog Working Group, David Harrington and Chris Lonvick, and the other members of the Working Group, in particular Alex Brown, Chris Calabrese, Steve Chang, Pasi Eronen, Carson Gaspar, Rainer Gerhards, Drew Gross, Albert Mietus, Darrin New, Marshall Rose, Andrew Ross, Martin Schuette, Holt Sorenson, Rodney Thayer, and the many Counterpane Internet Security engineering and operations people who commented on various versions of this proposal.

11. References

11.1. Normative References

[FIPS.186-2.2000] National Institute of Standards and Technology, "Digital Signature Standard", FIPS PUB 186-2, January 2000, <http://csrc.nist.gov/publications/ fips/archive/fips186-2/fips186-2.pdf>.

[FIPS.180-2.2002] National Institute of Standards and Technology, "Secure Hash Standard", FIPS PUB 180-2, August 2002, <http://csrc.nist.gov/publications/ fips/fips180-2/fips180-2.pdf>.

[RFC2119]

Bradner, S., "Key words for use in RFCs to Indicate Requirement Levels", BCP 14, RFC 2119, March 1997.

[RFC 4648 ]

Josefsson, S., "The Base16, Base32, and Base64 Data Encodings", RFC 4648, October 2006.

[RFC4880]

[RFC5226]

Callas, J., Donnerhacke, L., Finney, H., Shaw, D., and R. Thayer, "OpenPGP Message Format", RFC 4880, November 2007 .

Narten, T. and H. Alvestrand, "Guidelines for Writing an IANA Considerations section in RFCs", BCP 26, RFC 5226, May 2008.

[RFC5280]

[RFC5424] Cooper, D., Santesson, S., Farrell, S., Boeyen, S., Housley, R., and W. Polk, "Internet X.509 Public Key Infrastructure Certificate and Certificate Revocation List (CRL) Profile", RFC 5280, May 2008 .

Gerhards, R., "The syslog Protocol", RFC 5424, March 2009 . 
[RFC5425]

[RFC5 426]

Miao, F., Yuzhi, M., and J. Salowey, "TLS Transport Mapping for syslog", RFC 5425, March 2009.

Okmianski, A., "Transmission of syslog Messages over UDP", RFC 5426, March 2009.

11.2. Informative References

[NIST800.90]

National Institute of Standards and Technology, "NIST Special Publication 800-90: Recommendation for Random Number Generation using Deterministic Random Bit Generators", June 2006, <http:// csrc.nist.gov/publications/nistpubs/800-90/ SP800-90revised_March2007.pdf>.

[RFC3339] Klyne, G. and C. Newman, "Date and Time on the Internet: Timestamps", RFC 3339, July 2002.

[RFC3414]

Blumenthal, U. and B. Wijnen, "User-based Security Model (USM) for version 3 of the Simple Network Management Protocol (SNMPv3)", RFC 3414, December 2002 .

[RFC 4086 ]

Eastlake, D., Schiller, J., and S. Crocker, "Randomness Recommendations for Security", RFC 4086, June 2005.

Authors' Addresses

John Kelsey

NIST

EMail: john.kelsey@nist.gov

Jon Callas

PGP Corporation

EMail: jon@callas.org

Alexander Clemm

Cisco systems

EMail: alexecisco.com 